\title{
CONGELACIÓN DE ALIMENTOS
}

Ricardo Carranza'

\section{ASPECTOS FÍSICOS}

El agua es el mayor componente de los materiales alimenticios; al enfriar alimentos por debajo de $0 \mathrm{C}$, ocurre formación y hielo; esto empieza entre 0 y $-3 \mathrm{C}$ dependiendo de la concentración molar de los componentes celulares solubles. A medida que la temperatura baja progresivamente, más agua se convierte en hielo y el calor latente de formación del hielo se adiciona al calor sensible involucrado en enfriar ambos, hielo y la parte no congelada. Esto conduce a grandes variaciones en las capacidades calóricas, mientras que las conductividades térmicas cambian también considerablemente debido a que la conductividad del hielo es casi 4 veces mayor que la del agua.

Para la mayoría de los materiales biológicos la parte más importante del proceso de congelamiento tiene lugar en el intervalo de temperaturas entre -1 y $-8 \mathrm{C}$, mientras que las variaciones más grandes de capacidad calórica ocurren entre -1 C y -3 C. Sólo a temperaturas en el rango de -20 a $-40 \mathrm{C}$ y menores, no hay más cambios medibles con la temperatura en la cantidad de hielo presente y el agua remanente, si hay alguna, puede considerarse no congelable. Sin embargo, con propósitos prácticos, puede definirse un límite inferior para el intervalo de cambio de fase, en base a la relación de hielo al contenido total de agua de, digamos, $90 \%$.

Esta selección, además de constituir un criterio fácilmente aplicable, permite aproximar las curvas de capacidad calórica y conductividad térmica por encima y debajo de la zona de cambio de fase por medio de valores constantes. En la zona de cambio de fase puede usarse un triángulo y una línea recta para interpolar la capacidad calórica y la conductividad térmica (fig.1). Para materiales alimenticios homogéneos se obtienen valores

\section{Ingeniero pesquero}

FIGURA N $N^{\circ}$ 01: Estimulación de la capacidad calórica y $\mathrm{K}$ en problemas de congelación.

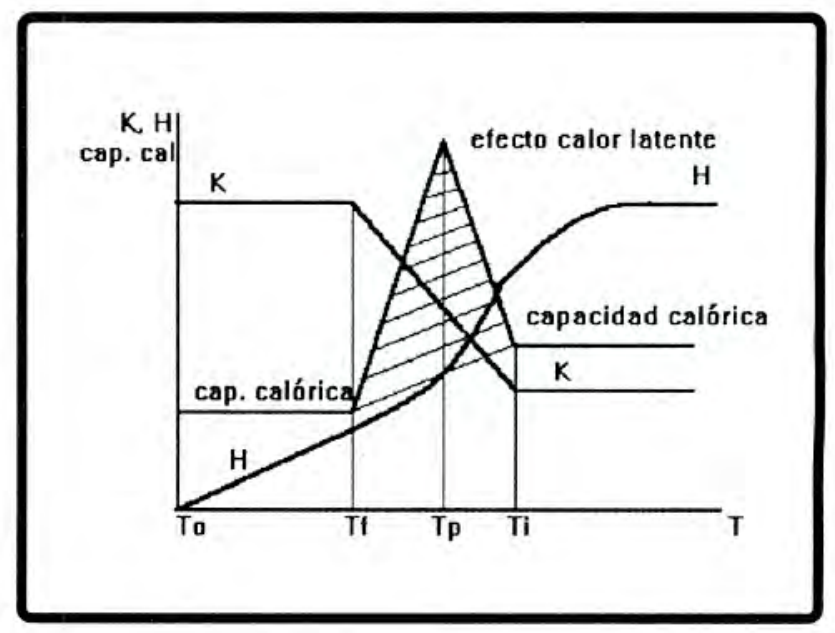

aproximados de las propiedades térmicas por encima y debajo de la congelación mediante las fórmulas:

c SUB L = p SUB SWL $\sim+\sim(1-p)$ c SUB d

K SUB L $\sim=\sim p K$ SUB $\{W L\} \sim+\sim(1-p) K$ SUB d

c SUB $s \sim=\sim p c$ SUB $\{W L\} \sim+\sim(1-p) c$ SUB $d$

K SUB $s \sim=\sim p K$ SUB $\{W L\} \sim+\sim(1-p) K$ SUB d

Una buena selección de valores para estas propiedades son:

$c_{W L}=4187 \mathrm{~J} / \mathrm{KgK}$

$c_{W s}=2093 \mathrm{~J} / \mathrm{KgK}$

$$
\mathrm{K}_{\mathrm{WL}}=0.59 \mathrm{~W} / \mathrm{mK}
$$

$c_{d}=1256 \mathrm{~J} / \mathrm{KgK}$

$\mathrm{K}_{\mathrm{ws}}=2.44 \mathrm{~W} / \mathrm{mK}$

$\mathrm{K}_{\mathrm{d}}=0.26 \mathrm{~W} / \mathrm{mK}$ así:

El efecto del calor latente puede evaluarse

lambda $\sim=\sim$ p lambda SUB $W \sim$; $\sim \sim$ lambda SUB $\mathrm{W} \sim=335 \mathrm{KJ} / \mathrm{Kg}$

donde $335 \mathrm{KJ} / \mathrm{Kg}$ es el calor de fusión/solidificación del agua. 
Luego, puede usarse álgebra simple para calcular el pico de la capacidad calórica si $T_{i}, T_{p}$ y $T_{\text {, }}$ son conocidas.

Usualmente es bastante seguro asumir $\mathrm{Ti}=$. $1 \mathrm{C}, \mathrm{Tp}=-3 \mathrm{C}$ y estimar Tf como el valor que da mayor ajuste entre valores de temperatura experimentales y computados.

Se han ensayado formas diferentes para las curvas de interpolación y valores diferentes para las temperaturas de referencia, pero las mejoras obtenidas, si hay alguna, no justifican complicaciones adicionales.

\section{VELOCIDAD DE CONGELACIÓN}

La calidad y cantidad de alimentos congelados están influenciados por factores relacionados a la velocidad de congelación, así, varias características de calidad, reciben influencia del tamaño de cristal que, a su vez, es función de la velocidad de congelación y la capacidad de producción de un sistema es directamente dependiente de la velocidad de remoción de la energía térmica de los productos, aunque el tiempo de congelación es el parámetro de diseño de interés directo.

La definición oficial de la velocidad de congelación, tal como la publicó el Instituto Internacional de Refrigeración (IIR) en 1971 es:

"La velocidad de congelación de una masa alimenticia es la relación entre la distancia mínima desde la superficie al centro térmico y el tiempo transcurrido desde que la superficie alcanza 0 C hasta que el centro térmico llegue a 5 C por debajo de la temperatura inicial de formación de hielo en el centro térmico; el espesor se mide en cm y el tiempo en $\mathrm{hr}$ y la velocidad de congelación se expresará en $\mathrm{cm} / \mathrm{hr}$ ".

\section{TIEMPO DE CONGELACIÓN - SU PREDICCIÓN}

La definición del IIR tiende a proporcionar una velocidad promedio basada en el cambio de temperatura en la masa del producto. Aunque la velocidad expresada por esta definición debería ser aceptable para la evaluación de las características de la calidad, el parámetro tiene poco uso en el diseño de procesos. A fin de incorporar la velocidad de congelación al diseño de procesos, debe expresársele como tiempo de congelación. Este parámetro puede convertirse directamente a tiempo de residencia del producto en el medio congelante. Además, el tiempo de congelación es necesario para el cálculo de la velocidad a la cual se requiere la refrigeración para aparejar la capacidad del sistema de congelación.
La predicción de los tiempos de congelación, es el punto de partida en el diseño de sistemas de congelación. Sin capacidad de predicción, el proceso completo de diseño se vuelve dependiente de experimentos de laboratorio y escala piloto. Aunque las etapas experimentales no pueden eliminarse, su magnitud puede reducirse significativamente.

\section{MÉTODOS DE PREDICCIÓN}

En general los métodos de predicción del tiempo de congelación pueden dividirse en 2 grupos: analíticos y numéricos; una revisión no exhaustiva es la que sigue:

\section{ANALITICOS}

Usualmente conducen a una o más expresiones para el cálculo del tiempo de congelación. Los más conocidos son: Ede(1949) es:

Plank (1913) cuya ecuación adaptada por t SUB F $\sim=\{$ rho lambda $\}$ OVER $\{$ T SUB i - T SUB INF $\}[\{\mathrm{Pa}\}$ OVERSM $\{\mathrm{h}\} \sim+\sim\{\mathrm{Ra}$ SUP 2$\}$ OVERSM $\{\mathrm{K}\}$ ]

Las limitaciones documentadas de esta ecuación son:

1) Los valores usados para el calor latente de fusión (lambda) deben estimarse y usualmente se aproximan a partir del contenido de humedad del producto y el calor latente de fusión del agua.

2) El tiempo requerido para la remoción del calor sensible por encima de la temperatura inicial de congelación (Ti) no es tomado en cuenta.

3) La temperatura final del producto congelado no aparece en la ecuación, indicando que el tiempo necesario para la reducción del calor sensible del producto congelado no se toma en cuenta.

4) La selección de un valor de conductividad térmica (K) para el producto congelado es difícil si se considera que esa propiedad es dependiente de la temperatura.

Pese a lo anterior, la simplicidad de la ecuación de Plank la mantiene como la más popular de las ecuaciones analíticas de predicción.

Nagaoka y colaboradores (1955) mejoran la ecuación de Plank incluyendo coeficientes experimentales que consideran el calor sensible por encima y debajo de la temperatura inicial de congelación. 
Cleland y Earle (1977;1979a y b) presentan la ecuación de Plank en la forma:

F SUB o = P P $[1$ OVER $\{B$ SUB i . S SUB $\{$ te $\}\}]+R$ [1 OVER S SUB $\{$ te $\}$ ]

Donde: $P=f(S t e, B i, P k)$ y $R=f(S t e, P k)$

$P$ y $R$ contienen coeficientes para esferas determinados por los autores usando la sustancia de prueba Karlsruhe. Los mismos autores en 1982 presentaron un análisis simplificado para una variedad de formas de productos.

Tao (1967) y Joshi y Tao (1974) presentaron una forma diferente de enfoque analítico basado en la solución numérica de las ecuaciones de conducción de calor con resultados presentados en forma de números adimensionales y cartas. Una de las limitaciones del método es que se necesitan valores discretos de las propiedades cuando los valores reales varían con la temperatura durante la congelación.

Mott (1964) presentó un trabajo similar al de Tao y Joshi en las bondades y limitaciones; el método usa 4 números adimensionales y valores de propiedades determinados experimentalmente, incluyendo la temperatura cuando $60 \%$ del agua está congelada. Requiere también valores discretos de propiedades y los valores sugeridos corresponden a medidas experimentales para un conjunto de productos especificos.

Hay varias otras modificaciones a la ecuación de Plank que tratan básicamente de la mejora en la estimación del calor latente de fusión y la forma del producto, entre ellos Levy (1958), Mellor (1976) y Gutschmidt (1964).

\section{NUMÉRICOS}

Durante los últimos 27 años han aparecido numerosas aplicaciones numéricas al problema de la congelación de alimentos; hoy se cuenta con formulaciones en Diferencias Finitas (DF) y Elementos Finitos (EF). En cuanto a datos experimentales, virtualmente todos ellos son para materiales de formas regulares y homogéneos, En estos casos EF no tiene ventajas sobre DF. Para estudiar el grado de precisión alcanzable con un método de DF es necesario primero reestablecer la base del método. En el caso de una placa infinita (fig.2), un balance de calor sobre una pequeña sección de ella da:

(Mc $\{$ PARTIAL T\} OVERSM \{PARTIAL $t\})$ SUB $n=(K A\{$ PARTIAL T\} OVERSM \{PARTIAL X\}) SUB $\{n+1 / 2\}-(K A\{$ PARTIAL T\} OVERSM \{PARTIAL X\}) SUB $\{n-1 / 2\}$
FIGURA $N^{\circ}$ 02: llustración esquemática de un pequeño elemento de espesor $\square x$ dentro de una placa infinita de sección transversal A.

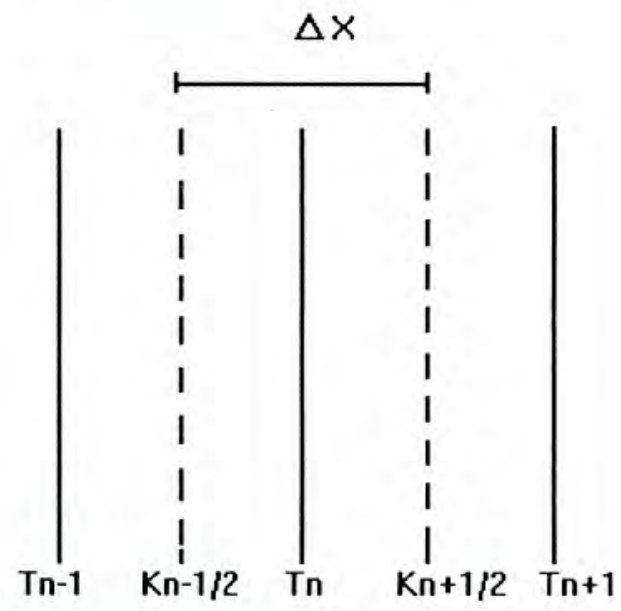

Esta ecuación asume que el material dentro del enésimo elemento, tiene una masa $M$, volumen $A$ $X$, calor especifico $c$ y puede ser representado por una única temperatura $\mathrm{Tn}$. La capacidad calórica específica para esta región se evalúa a Tn. Los flujos de conducción de calor se evalúan en posiciones $(n+1 /$ 2) y $(n-1 / 2)$. La ecuación general de flujo de calor se deriva de la ecuación ( 8 ) haciendo $M=A X$ (densidad) y reacomodando:

( rho c $\{$ PARTIAL T\} OVERSM \{PARTIAL $t\}$ ) SUB $n \sim=\sim 1$ OVERSM $\{$ DELTA $X\}$ [(K \{PARTIAL T\} OVERSM \{PARTIAL X\}) SUB $\{n+1 / 2\} \sim \sim(K\{$ PARTIAL T\} OVERSM \{PARTIAL X) $S \cup B\{n-1 / 2\}]$

que en el límite cuando $X$---> 0 se vuelve:

rho $c$ PPARTIAL T\} OVERSM \{PARTIAL $t\} \sim=\sim$ PARTIAL OVERSM \{ PARTIAL X\} (K \{PARTIAL T\} OVERSM \{ PARTIAL X\})

que a menudo se escribe:

$\{$ PARTIAL T\} OVERSM $\{$ PARTIAL $t\} \sim=\sim$ alpha $\{$ PARTIAL SUP 2 T\} OVERSM \{PARTIAL X SUP 2\}

que es verdadera sólo si $\mathrm{K}$ es independiente de la posición en el material; es decir, si el material es homogéneo. Si la temperatura varia con la posición y $\mathrm{K}$ con la temperatura, $\mathrm{K}$ no puede ser movida del corchete en la ecuación (10) y la (11) no es válida. Otra alternativa es:

\{PARTIAL T\} OVERSM \{PARTIAL $t\} \sim=\sim$ PARTIAL OVERSM \{PARTIAL X\} [ alpha \{PARTIAL T\} OVERSM \{ PARTIAL X\}] 
para que á sea transferida al término diferencial del lado derecho (ecuación (12)), aunque la ecuación (8) sea aún correcta, la capacidad calórica no debe variar con la posición es decir:

rho $c$ SUB $\{n+1 / 2\} \sim=\sim$ rho $c$ SUB $\{n\} \sim=\sim$ rho $\operatorname{SUB}\{n-1 / 2\}$

La temperatura puede variar con la posición, y la implicancia es entonces que la ecuación (12) se aplica solamente si la capacidad calórica no es variable con la temperatura aunque $\mathrm{K}$ pueda variar.

\section{LA RELACIÓN Á - T : ALGO DE HISTORIA}

La difusividad térmica (á) es dependiente de la temperatura:

$$
\text { (13)alpha }(T) \sim \sim\{K(T)\} \text { OVER }\{\text { rho }(T) . c S U B\{a p\}(T)\}
$$

La relación entre la difusividad térmica (á) y la temperatura $(T)$ es singular para productos alimenticios y sus caracteristicas representan un reto significativo en la obtención de soluciones numéricas a la ecuación (12). Un ejemplo de esta relación predicha por métodos debidos a Heldman (1982) aparece en la fig. 3 .

Han habido 3 enfoques destinados a obtener soluciones numéricas a las ecuaciones de conducción de calor durante la congelación de alimentos:

1) El uso de funciones separadas de calor específico y calor latente en lugar de una función de calor específico aparente (Cap).

2) El uso de una relación á - T aproximada para lograr facilidad en la solución.

FIGURA N ${ }^{\circ}$ 03: Relación entre difusividad térmica y temperatura durante congelación de productos alimenticios tal cual pronosticada con asunción de temperatura inicial de congelación.

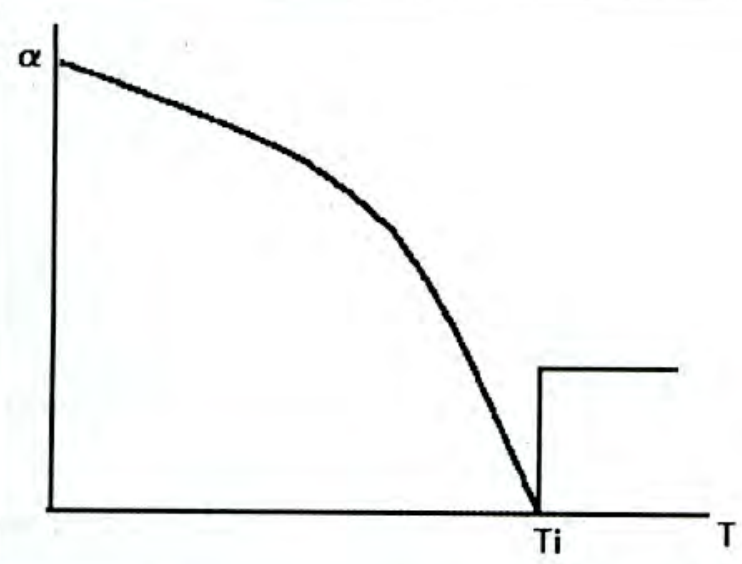

3) Solución de las ecuaciones diferenciales parciales usando una función á - T real.

La separación de las funciones de calor específico y latente requiere que todo el calor latente sea liberado a temperatura constante, tal como lo demostró Charm (1972). La aceptación de este análisis tiene dependencia significativa de la temperatura usada para la liberación del calor latente y requiere seleccionar valores discretos para las propiedades del producto congelado.

Otros investigadores han usado transformaciones matemáticas para obtener soluciones para valores de propiedades dependientes de la temperatura. En general estos análisis requieren de todos modos valores de propiedades del producto congelado como datos de entrada para los métodos de predicción.

Bonacini y Comini (1973) y Bonacini y colaboradores(1974) usaron una función matemática para aproximar la relación á - T para resolver variedades de situaciones y para varias formas geométricas de productos. La bondad de este análisis para predecir tiempos de congelación exactos está directamente relacionada a la exactitud de la relación mencionada comparada con la relación real. Esta última varía de un producto a otro y la capacidad de predicción variará con las características de los productos.

Heldman (1974b) y Heldman y Gorby $(1974 ; 1975 a, b)$ presentaron un trabajo en el que hacen uso de las características y propiedades del producto no congelado para predecir la relación á - T. Las ventajas incluyen aqui que no hay otra asunción que no sea el concepto de solución ideal requerida para predecir la relación agua no congelada - temperatura. Este análisis conduce a la predicción de todas las propiedades del alimento congelado que son necesarias para el proceso de congelación. La limitación básica es probablemente la disponibilidad de datos exactos de propiedades para alimentos no congelados.

Mannaperuma y Singh (1988) presentaron un trabajo en el que vía formulación explicita de DF que involucra formulación entálpica, evitan la fuerte discontinuidad experimentada cuando se usa la formulación del calor específico aparente.

Los métodos analíticos se usan para calcular valores únicos de tiempo que representan el tiempo requerido para la congelación del producto. Los 
métodos numéricos que implican simples o sofisticados programas computacionales basados en técnicas de DF y EF, predicen la historia térmica para cualquier posición dentro del producto.

ESQUEMAS NUMERICOS USADOS COMUNMENTE EN EL CALCULO DEL TIEMPO DE CONGELACION (caso placa infinita)

\section{Esquema de Lees}

(rho c) SUB n SUP i. $\{T$ SUB n SUP $\{i+1\}-T$ SUB n SUP $\{i-$ 1\}\} OVER $\{2$ DELTA $t\}=1$ OVER $\{3($ DELTA $X)$ SUP 2$\}$ LBRACE $\{K$ SUB $\{n+1 / 2\}$ [ ( T SUB $\{n+1\}$ SUP $\{i+1\}-T$ SUB $n$ SUP $\{i+1\})\}+(T$ SUB $\{n+1\}$ SUP i-T SUB $n$ SUP i)

$+(T$ SUB $\{n+1\} \operatorname{SUP}\{i-1\}-T$ SUB $n$ SUP $\{i-1\})]-K$ SUB $\{n-$ $1 / 2\}$ SUP i [(T SUB $n$ SUP $\{i+1\}-T$ SUB $\{n-1\}$ SUP $\{i+1\})$ $+(T$ SUB $n$ SUP i-T SUB $\{n-1\}$ SUP i $)+(T$ SUB $n$ SUP $\{i-1\}-$ T SUB $\{n-1\}$ SUP $\{i-1\})]$ RBRACE

Esquema modificado de Crank-Nicholson

( rho c) SUB $n$ SUP i. $\{$ T SUB $n$ SUP $\{i+1\}-T$ SUB $n$ SUP i $\}$ OVER $\{$ DELTA $t\}=1$ OVER $\{2$ (DELTA X) SUP 2$\}$ LBRACE $K$ SUB $\{n+1 / 2\}$ SUP i [(T SUB $\{n+1\}$ SUP $\{i+1\}-T$ SUB $n$ SUP $\{i+1\})+(T$ SUB $\{n+1\}$ SUP i $-T$ SUB $n$ SUP i) ] -

K SUB $\{n-1 / 2\}$ SUP i [ (T SUB $n$ SUP $\{i+1\}-T$ SUB $\{n-1\}$ SUP $\{i+1\})+(T$ SUB $n$ SUP i-T SUB $\{n-1\}$ SUP i) ] RBRACE

Esquema completamente implícito

( $r$ ho $c$ ) SUB $n$ SUP i . \{T SUB $n$ SUP $\{i+1\}-T$ SUB $n$ SUP i $\}$ OVER $\{$ DELTA $t\}=1$ OVER $\{($ DELTA $X)$ SUP 2$\}$ [ $K$ SUB $\{n+1 / 2\}$ SUP i(T SUB $\{n+1\}$ SUP $\{i+1\}-T$ SUB $n$ SUP $\{i+1\})-$ K SUB $\{n-1 / 2\}$ SUP i(T SUB $n$ SUP $\{i+1\}-T$ SUB $\{n-1\}$ SUP $\{i+1\})]$

Esquema completamente explícito

( rho c) SUB n SUP i. $\{T$ SUB a SUP $\{i+1\}-T$ SUB $n$ SUP i\} OVER $\{$ DELTA $t\}=1$ OVER $\{($ DELTA $X)$ SUP 2$\}$ [ $K$ SUB $\{n+1 / 2\}$ SUP i(T SUB $\{n+1\}$ SUP i-T SUB $n$ SUP i)-K SUB $\{n-1 / 2\}$ SUP i(T SUB $n$ SUP i-T SUB $\{n-1\}$ SUP i) ]

Esquema explicito de transformación de entalpia

$\{$ S SUB $n$ SUP $\{i+1\}-H$ SUB $n$ SUP i $\}$ OVER $\{$ DELTA $t\}=1$ OVER $\{2($ DELTA X) SUP 2$\}$ [ $K$ SUB $\{n+1 / 2\}$ SUP i(T SUB $\{n+1\}$ SUP i-T SUB $n$ SUP i)-K SUB $\{n-1 / 2\}$ SUP i(T SUB $n$ SUP i-T SUB $\{n-1\}$ SUP i) ]

donde $\sim$ H SUB $n$ SUP $\{i+1\} \sim y \sim T$ SUB $n$ SUP $\{i+1\} \sim$ SOn $\sim$

\section{REFERENCIAS BIBLIOGRÁFICAS}

HELDMAN D.R.,Factors influencing food freezing rates, Fd. Techn.(1983) 37(4), 103-109

REBELLATO L., DEL GIUDICE S., COMINI G., Finite Element analysis of freezing processes in foodstuffs, J. Fd. Sc. 43 (1978) $237-246$

MANNAPERUMA J., Prediction and thawing times of foods using a numerical method based on enthalpy formulation. J. Fd. Sc. vol $53 \mathrm{~N}^{\circ} 21988$ relacionadas $\sim$ luego $\sim$ de $\sim$ cada $\sim$ paso $\sim$ de $\sim$ tiempo

Esquema modificado de Crank-Nicholson usando á

$\{$ T SUB $n$ SUP $\{i+1\}-T$ SUB $n$ SUP i\} OVER $\{$ DELTA $t\}=1$ OVER $\{2(D E L T A X)$ SUP 2$\}$ LBRACE (K OVERSM \{ rho c\}) SUB $\{n+1 / 2\}$ SUP i [ (T SUB $\{n+1\}$ SUP $\{i+1\}-T$ SUB $n$ SUP $\{i+1\})+(T$ SUB $\{n+1\}$ SUP i-T SUB $n$ SUP i) $]-(K$ OVERSM $\{$ rho $c\})$ SUB $\{n-1 / 2\}$ SUP $i$.

[ ( T SUB $n$ SUP $\{i+1\}-T$ SUB $\{n-1\}$ SUP $\{i+1\})+(T$ SUB $n$ SUP i-T SUB \{n-1\} SUP i) ] RBRACE

\section{NOMENCLATURA}

$\begin{aligned} \mathrm{C} & =\text { capacidad calórica especifica }(\mathrm{J} / \mathrm{KgK}) \\ \mathrm{K} & =\text { conductividad térmica }(\mathrm{W} / \mathrm{mK}) \\ \mathrm{p} & =\text { fracción de masa de agua } \\ \mathrm{T} & =\text { efecto del calor latente }(\mathrm{J} / \mathrm{Kg}) \\ \mathrm{t} & =\text { temperatura }(\mathrm{C}) \\ \mathrm{P}, \mathrm{R} & =\text { densidad del producto }\left(\mathrm{Kg} / \mathrm{m}^{3}\right) \\ \mathrm{a} & =\text { dimensión caracteristica } \\ \mathrm{h} & =\text { coeficiente de transferencia de calor por } \\ & \text { convección } \\ \mathrm{Fo} & =\text { Wúmero de Fourier, át/a }{ }^{2} \\ \mathrm{Bi} & =\text { número de Biot, ha/K } \\ \mathrm{Ste} & =\text { número de Stefan, } \mathrm{C}_{\mathrm{s}}(\mathrm{Ti}-\mathrm{T}) / \mathrm{H} \\ \mathrm{H} & =\text { cambio en entalpia } \mathrm{en} \mathrm{la} \text { congelación } \\ \mathrm{Pk} & =\text { número de Plank, } \mathrm{C}_{\mathrm{L}}(\mathrm{T} 1-\mathrm{Ti}) / \mathrm{H} \\ \mathrm{a} & =\text { difusividad térmica }(\mathrm{m} 2 / \mathrm{s}) \\ \mathrm{X} & =\text { distancia variable }(\mathrm{m})\end{aligned}$

\section{Subindices}

$\begin{aligned} \mathrm{W} & =\text { agua } \\ \mathrm{L} & =\text { liquido } \\ \mathrm{d} & =\text { seco } \\ \mathrm{S} & =\text { sólido } \\ \mathrm{i} & =\text { inicial } \\ \mathrm{p} & =\text { pico } \\ \mathrm{f} & =\text { final } \\ \mathrm{F} & =\text { congelación } \\ \mathrm{ap} & =\text { medio congelante } \\ & =\text { aparente }\end{aligned}$

CLELAND A.C., EARLE R.L., Assessment of freezing time prediction methods, J. Fd. Sc. vol 49 (1984)

HELDMAN D.R., Computer simulation of food freezing processes, proced. IV Int. Congress Food Science and Techno logy (1974) vol IV $397-406$

CARRANZA R., Relationship between calculated freezing times and those measured in real food packs, MSc thesis University of Reading 1990 